\title{
High-extraction-efficiency Si-QD LED using rugged surface pattern
}

\author{
K.H. Kim, J.H. Shin, N.M. Park, T.Y. Kim, C. Hur, K.S. Cho, and G.Y. Sung \\ Electronics and Telecommunications Research Institute (ETRI) \\ 161 Gajeong-dong, Yuseong-gu, Daejeon, Korea
}

Currently, light emitting diodes (LEDs) are used for wide range of applications such as traffic signal, out-side huge advertising display, rear light for LCD or keypad of telephone, and lighting fixtures using compound semiconductor, involving of GaAs, InP, GaN. However, since some research groups were reported for Si-based light-emitting diode (Si-LED) ${ }^{1-4}$, the interesting of Si photonics has been increased. Of course, the brightness of Si-based LEDs is much lower than that of compound semiconductor based LEDs due to the intrinsic optical property of $\mathrm{Si}$, which is indirect band gap material. Although Si-based optoelectronic device has a poor optical property, it also has many advantages such as low cost, well developed CMOS technology, and effective integration.

In the case of Si based LEDs, we already reported that Si-QD LEDs have high external quantum efficiency and a good quantum confinement effect of nc-Si embedded in amorphous silicon nitride matrix ${ }^{3}$. From the report, we found a possibility of Si based LED. If more bright Si-LEDs are developed, the paradigm of photonic industry will be changed largely due to advantages of Si material. In this study, we will report more improved Si-LED using rugged surface pattern.

We calculate the extraction efficiency enhancement Si-LED using micron scale rugged surface pattern. In general, most of compound semiconductors LEDs have applied a sub-micron scale pattern to increase their extraction efficiency. Thus, we firstly applied micron scale surface pattern for Si based LED to improve extraction efficiency. We simply calculated by geometric optics, Snell's law. Snell's law indicates relationship of refraction and reflection between two different media and it is determined an escape angle of LED. Usually, there is a significant difference between the internal efficiency of LEDs and their external efficiency. Ordinary LEDs have a few percent external efficiencies due to low extraction efficiency. The reason for this low value is the difficulty for light to escape from high refractive index semiconductors. To calculate more simply, we used the 1D model, which has periodic square pattern. Figure 
1 is a schematic diagram of light extraction. We assumed that repeated line light source is separated several distances of $\mathrm{h} 2$ from the surface and $\mathrm{h} 1$ is height of pattern. If the refractive index of $\mathrm{SiC}$ layer, transparent hole doping layer, is an about 2, the escape cone for internal light in Si-QD LED is only $\sim 30^{\circ}$. The light which fails to emit from the LED is absorbed by semiconductors and electrodes. To overcome this problem, we deposited transparently thick layer, $\mathrm{SiN}$, which has a similar refractive index of $\sim 2$, on $\mathrm{SiC}$ layer and then, formed periodically rugged structures using etching process. Figure 2 shows the 1-D simulation results of the extraction probability for the rugged surface structure. Figure 2(a) shows that the detection probability of emitted light increases as height of mesa pattern increases. Figure 2(b) also shows the ratios of pattern size and period. There is an optimum ratio of 0.6 for 4 um pattern at refractive index of 2 and the height of h1 and h2 of $1 \mathrm{um}$, respectively. Maximum enhancement of the extraction probability was about $60 \%$.

It is very important to determine an etching condition for rugged surface pattern formation. We used reactive ion etching (RIE) technique to make a very steep wall of rugged pattern. From the figure 3, we clearly observed very steep pattern of SiN and very smooth Si surface and SiN surface. We found optimum etching condition, which is a good shape and minimum ion damage at the same time, from the many experimental results. Etching conditions: etching power of $100 \mathrm{~W}$, self-bias voltage of $230 \mathrm{~V}$, etching gas of $\mathrm{CF}_{4}: \mathrm{Ar}=20: 5 \mathrm{sccm}$, working pressure of $30 \mathrm{mTorr}$, etch rate of $20 \mathrm{~nm} / \mathrm{min}$.

Using the theoretical results and optimum etching conditions, we naturally fabricated Si-QD LED, involving rugged surface pattern. We firstly measured electrical property from the Si-QD LED, involving rugged surface pattern but we could not obtain electroluminescence due to the metallization problem. So, we continually fabricated SiQD LEDs and are measuring property of them at present. In the conference, we will report experimental results and compare with others. And we will also discuss differences between experimental and theoretical results from electroluminescence of Si-QD LEDs. 


\section{Reference}

1. M.L. Brongersma, A. Polman, K.S. Min, E. Boer, T. Tambo, and H. A. Atwater, Appl. Phys. Lett., 72, 2577, 1998.

2. N.M. Park, T.S. Kim, and S.J. Park, Appl. Phys. Lett., 78, 2575, 2001.

3. K.S. Cho, N.M. Park, T.Y. Kim, K.H. Kim, and G.Y. Sung, Appl. Phys. Lett., 86, $071909,2005$.

4. T.Y. Kim, N.M. Park, K.H. Kim, Y.W. Ok, T.Y. Seong, C.J. Choi, and G.Y. Sung, Appl. Phys. Lett., 85, 5355, 2004.

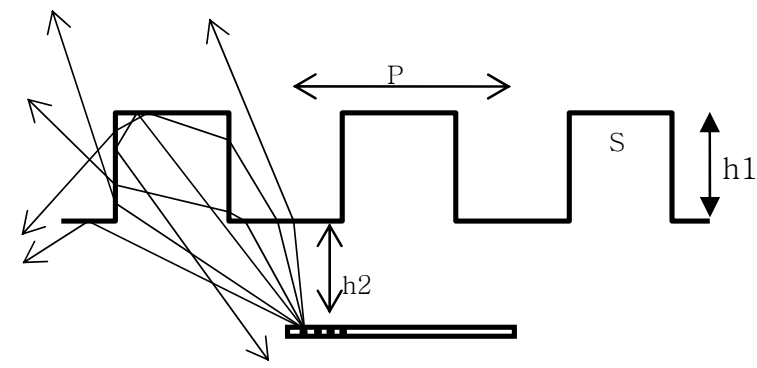

Fig. 1 Schematic diagrams of rugged surface structure of Si-LED and trajectory of emitted light from the light source.
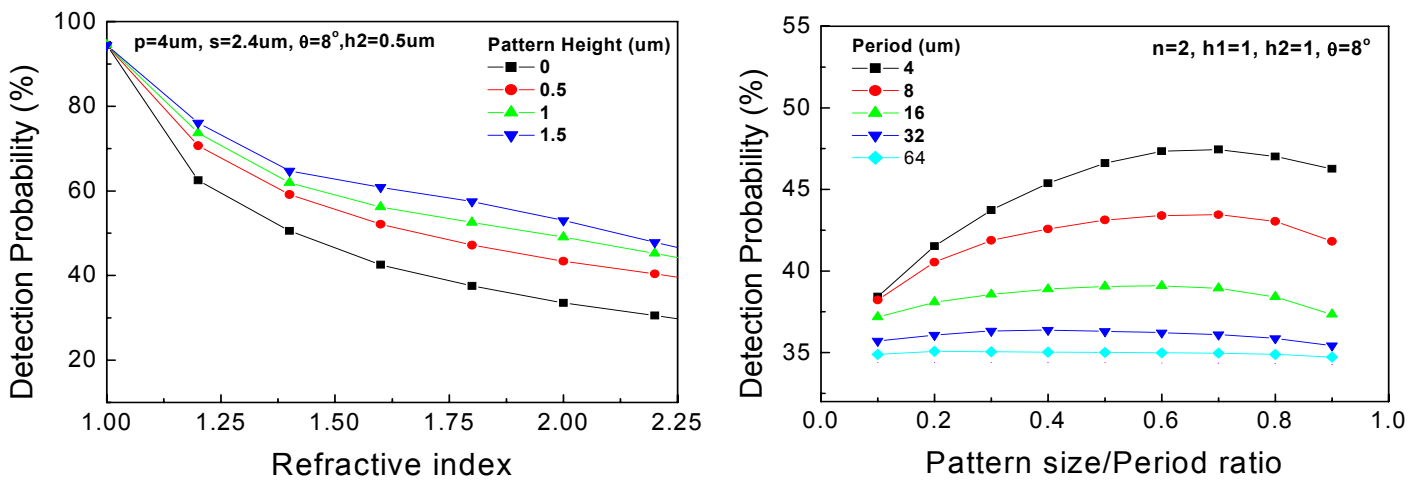

Fig. 2 The probabilities of detected light at the function of various refractive indexes and the ratio of the pattern height and periodicity.

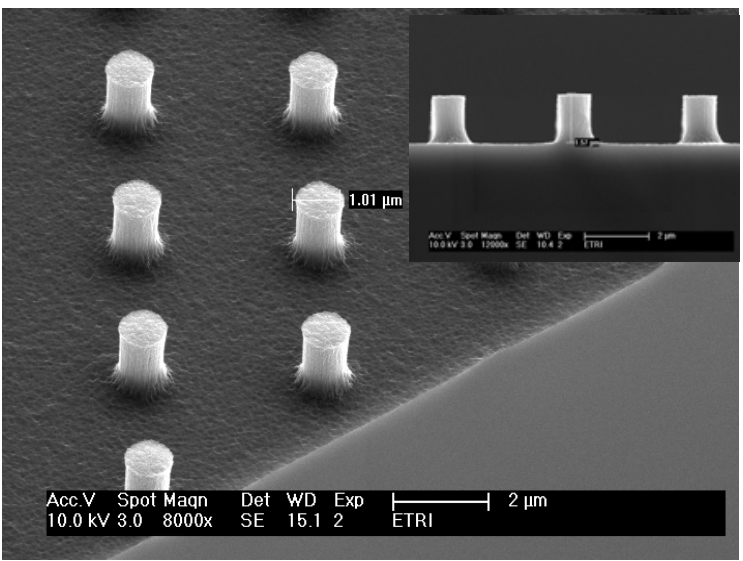

Fig. 3 The SEM image of etched SiN rugged pattern layer, which is made by reactive ion etching (RIE). Inset shows cross sectional image of $\mathrm{SiN}$ steep pattern. 